\title{
Visuo-vestibular interaction in the reconstruction of travelled trajectories.
}

\author{
R.J.V. Bertin, A. Berthoz
}

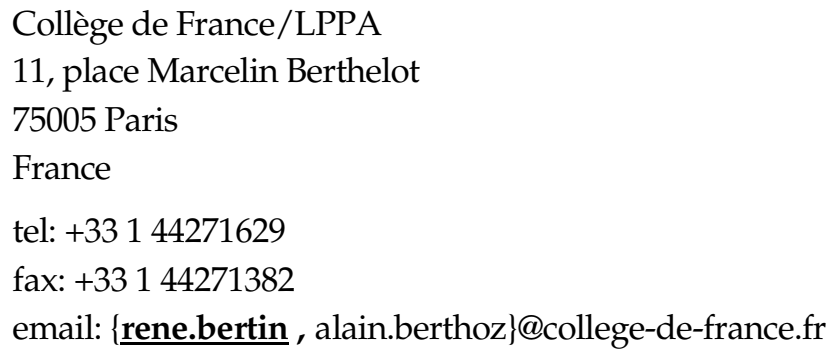

email: $\{\underline{\text { rene.bertin , alain.berthoz }\} @ c o l l e g e-d e-f r a n c e . f r}$ 


\title{
Visuo-vestibular interaction in the reconstruction of travelled trajectories.
}

\author{
R.J.V. Bertin, A. Berthoz
}

\begin{abstract}
We recently published a study of the reconstruction of passively travelled trajectories from optic flow. Perception was prone to illusions in a number of conditions, and not always veridical in the others. Part of the illusionary reconstructed trajectories could be explained by assuming that subjects base their reconstruction on the ego-motion percept built during the stimulus' initial moments. In the current paper, we test this hypothesis using a novel paradigm: if the final reconstruction is governed by the initial percept, providing additional, extra-retinal information that modifies the initial percept should predictably alter the final reconstruction. The extra-retinal stimulus was tuned to supplement the information that was under-represented or ambiguous in the optic flow: the subjects were physically displaced or rotated at the onset of the visual stimulus. A highly asymmetric velocity profile (high acceleration, very low deceleration) was used. Subjects were required to guide an input device (in the form of a model vehicle; we measured position and orientation) along the perceived trajectory. We show for the first time that a vestibular stimulus of short duration can influence the perception of a much longer lasting visual stimulus. Perception of the ego-motion translation component in the visual stimulus was improved by a linear physical displacement: perception of the ego-motion rotation component by a physical rotation. This led to a more veridical reconstruction in some conditions, but to a less veridical reconstruction in other conditions.
\end{abstract}

\section{Introduction}

There is an ongoing debate about the relative importance of different sensory systems in the perception and control of ego-motion, and the interactions between these modalities. It has long been thought that navigation and orientation were mainly subserved by visual ego-motion perception. In particular, the direction of heading - where we are going - was supposed to be retrieved from the optic flow (Gibson, 1950; Koenderink, 1986; Lappe, Bremmer et al., 1999) and/or from other visual sources of information (Rushton, Harris et al., 1998). A renewed interest in the role and function of other sensory modalities in the perception of ego-motion, orientation and navigation - often in relation to virtual reality - is supplying evidence that the visual system is less dominant than thought.

It is well established that humans can perceive their heading direction from optic flow patterns, with high accuracy and almost instantaneously. It is equally well established that illusions can occur, inducing large errors. A famous example of such an ambiguous optic flow field is the flow that represents a straight trajectory, but that is perceived as a curved path (Royden, Crowell et al., 1994; Crowell, Banks et al., 1998; Wann, Swapp et al., 2000; Li and Warren, Jr., 2000). This optic flow stimulus simulates an observer moving along a straight line while making an eye- or head movement, e.g. to fixate an object at eye-height to the side of the path. It is generally thought that the illusion occurs because information is missing from, or under-represented in, the optic flow. Infor-

Visuo-vest. interaction in ego-motion reconstruction $\quad$ René JV Bertin $2 / 24$


mation can be added via several sensory modalities to disambiguate this stimulus. When the observer actively makes an eye- or head movement corresponding to the one simulated, the illusion disappears and heading perception becomes more veridical (Crowell, Banks, Shenoy, and Andersen, 1998) - but not when the subject is full-body rotated passively. The illusion can also be made to disappear by increasing the amount of visual information available, for instance by presenting a structured visual environment (Li and Warren, Jr., 2000) instead of a virtual landscape consisting only of white dots, or even by adding a single landmark to such a "dotscape" (Bertin and Israël, unpublished).

Other authors have studied the perception of travelled distance and/or rotation, focussing on the contributions from the visual, vestibular and somatosensory systems (Bakker, Werkhoven et al., 1999; Mergner and Becker, 1990; Hlavacka, Mergner et al., 1996; Mergner, Schweigart et al., 2000; Harris, Jenkin et al., 2000; Loose, Ayan et al., 1999; Probst, Loose et al., 1996; Probst, Loose et al., 1995; Mesland, 1998). These studies clearly indicate that non-visual systems play an important role in ego-motion perception, although it is not yet quite clear which role. One study (Bakker, Werkhoven et al., 1999) found a systematic undershoot in turning through an instructed angle (overestimation of perceived ego-velocity) with better performance when vestibular and kinaesthetic information was used versus when only visual information was available. Another study (Groen, Valenti Clari et al., 2000) found overestimation of the vestibular stimulus in simulators (cf. Pavard and Berthoz, 1977 and Buizza, Leger et al., 1980), and mentioned systematic underestimation of visually perceived ego-velocity (that can also occur when real-world vestibular and kinaesthetic information is available, when driving a car via a video-link!). Harris and co-workers (Harris, Jenkin et al., 2000; Redlick, Jenkin et al., 2001) found undershoot of the instructed distance when providing only a visual display consisting of properly scaled representations of well-known environments. Jürgens (Jürgens, Boß et al., 1999) found a range effect (undershoot of large rotations, overshoot of small rotations) when subjects were to estimate rotations using only vestibular information. One suggestion emerging from many of these studies is that the vestibular system dominates the visual system, at least in simulators (e.g. Groen, Valenti Clari et al., 2000; Harris, Jenkin et al., 2000; Redlick, Jenkin et al., 2001), with instructed distances being undershot when only visual information is available, but (slightly) overshot when the corresponding vestibular information is also provided. Other studies have suggested the possibility of a "max rule" where the brain "listens to" the sensory system indicating the biggest travelled distance or turned angle (von der Heyde, Riecke et al., 2001; Lambrey, Viaud-Delmon and Berthoz, 2002).

In an earlier series of experiments, we studied the reconstruction of passively travelled manoeuvres in the plane; from vestibular information alone (Ivanenko et al., 1997) and from optic flow information alone (Bertin, Israël et al., 2000). Here, we use the term manoeuvre to designate a specific trajectory (path) combined with a specific orientation (yaw) at any given point along that trajectory, orientation need not be yoked to the path. We simulated manoeuvres by displacing a virtual observer through a virtual environment; when the resulting optic flow in the virtual observer's field of view is presented to a (human) observer, this can create the sensation of passive travel ("vection"), ideally along that simulated manoeuvre. Among the simulated manoeuvres that we presented, several generated flow fields known to be ambiguous. We included the straight path + yaw (linear+yaw) manoeuvre mentioned above. Until recently, this illusion had been shown only for short stimulus durations, simulating relatively small rotation of the eye or head (not more than a few degrees). We simulated much larger subject rotations: between 90 and 360 degrees. Nevertheless, the illusion still occurred: the majority of the subjects perceived themselves to be travelling along a circular trajectory, with orientation more or less (tangentially) fixed relative to that path. That is quite an impressive illusion! Somehow, it had gone unnoticed that all these displays contained a phase of sideways motion, in most cases evolving into backward motion, and so forth.

Visuo-vest. interaction in ego-motion reconstruction $\quad$ René JV Bertin $3 / 24$


This illusion is often explained by a strong resemblance between this kind of stimulus, and the flow corresponding to the illusory, perceived manoeuvre (e.g. Royden, Crowell, and Banks, 1994, but cf. Kim and Turvey, 1998 and Wann and Schwapp, 2000). This resemblance between the two flows indeed exists, but is easy to see that in our stimuli it cannot last longer than a fraction of the duration, as much larger rotations are simulated (see also Ehrlich, Beck et al., 1998).

How then, could one explain the fact that the illusion still occurs? We propose that the initial percept is of crucial importance. Imagine an ideal subject: naive and inexperienced, but one intent on being efficient in the required task (like any observer!). It is conceivable that he or she would update an existing, robust ego-motion percept - e.g. the initial percept - only when some qualitative change occurs in the stimulus. In between such events, they would then simply extrapolate that established percept. Such events do not occur in our case. Therefore such a strategy would explain our result, because, in the linear+yaw stimuli, the initial percept certainly is the illusory perception of a circular path. Extrapolation of that percept would yield the responses we found.

Thus, our hypothesis is that the initial percept is important in ego-motion perception tasks involving the reconstruction of travelled trajectories. One can then address the question if it is possible to modify the final percept by altering the initial impression. During the initial stimulus phases, one could supplement the information that is ambiguous or under-represented in the optic flow, for instance by physically displacing the subjects at stimulus onset. If this extra, non-retinal information improves the initial percept's veracity, then the final percept (the reconstructed manoeuvre) should be more veridical too if indeed it is determined by the initial percept. The additional information should be present only at the stimulus onset, thus calling for an impulse-like vestibular stimulus: high acceleration followed by sub-threshold deceleration.

In this paper, we report on a study that addressed this question. We selected 3 different manoeuvres from our previous experiments, and presented them at different scales in an experimental protocol that closely followed the protocol of our earlier studies. One of these was the linear+yaw manoeuvre (linear 180-turn) discussed at length above. The other 2 were both semicircles. Semicircular manoeuvres with tangential observer orientation (observer "looking forward in the direction of movement": semicircle forward) had often been perceived as rotations in place, especially for a small radius (undershoot of the translation component). Semicircular manoeuvres with outward observer orientation (observer oriented with the back towards the circle's centre; semicircle outward) had often been perceived as lateral, linear translation, especially for a large radius (undershoot of the rotation component). To test our hypothesis, we presented the stimuli purely visually, and together with an initial vestibular stimulus designed to supplement the information in the visual stimulus. The vestibular stimulus consisted of either purely linear motion, or purely angular motion, but never a combination of the two. Whereas this choice possibly induced mild visuo-vestibular conflict, it had the advantage that it excited either the linear or the angular vestibular acceleration sensors but not both. It also provided a means to add supplementary information that was minimal and controllable to the best possible extent (e.g. interactions between semicircular canals and otoliths were avoided). Strongly conflicting stimulus combinations were avoided.

\section{Materials and Methods}

\section{Subjects}

A total of 14 subjects participated in this study, 8 males and 6 females. All subjects were in their early twenties to mid-thirties, and gave their informed consent before the experiment was run. Of 
this population, 9 subjects participated in a purely visual pilot experiment that took place several weeks to several months before the visuo-vestibular experiment. They had varying degrees of experience with psychophysical experiments, but all were naïve as to the purposes of the current experiment. The experimental protocol was approved by the local ethics committee following national ethics guidelines and conformed to the Helsinki Convention.

In each trial, a visual stimulus was presented that showed the optic flow corresponding to a passive (simulated) manoeuvre. In $50 \%$ of the trials, this visual stimulus was complemented by a physical displacement that generated a vestibular stimulation. We will first describe the vestibular stimuli.

\section{Figure 1 ABOUT HERE.}

\section{Apparatus: physical displacements}

For the physical displacements, we used a mobile robot, the Robuter ${ }^{\mathrm{TM}}$ (Robosoft, Bayonne, France): figure 1. This is a remotely controlled moving platform, onto which a racing car seat has been mounted (Berthoz et al., 1995). The best way to present an impulse-like vestibular stimulus with this apparatus was to use a highly asymmetric velocity profile, quickly attaining a maximum velocity, and slowly decelerating to standstill. Standstill was programmed to occur after the end of the visual stimulation, in order to avoid a "stop signal" during the visual stimulus, as would be created by a too sudden deceleration or even because of the brakes that the robot applies at the end of any movement. The initial acceleration was maximised such that the following deceleration was the lowest and "smoothest" possible, with the smoothest possible transition from acceleration to deceleration. Linear movements were thus presented that attained a maximum velocity of around $0.33 \mathrm{~m} / \mathrm{s}$ at $\mathrm{t} \approx 0.8 \mathrm{~s}$ (acceleration of $0.87 \mathrm{~m} / \mathrm{s}^{2}$ attained after $0.5 \mathrm{~s}$; deceleration of $0.025 \mathrm{~m} / \mathrm{s}^{2}$, total displacement of about $1 \mathrm{~m}$ ). Angular movements attained a maximum of around $\pm 23^{\circ} / \mathrm{s}$ at $\mathrm{t} \approx 1 \mathrm{~s}$ (acceleration of around $\pm 54^{\circ} / \mathrm{s}^{2}$ attained after $0.73 \mathrm{~s}$, deceleration of around $\pm 2^{\circ} / \mathrm{s}^{2}$, total rotation slightly under $\left.\pm 90^{\circ}\right)^{1}$. Figures $2 \mathrm{a}$ and $2 \mathrm{~b}$ show the average physical velocity profiles, as determined off-line from the odometric data recorded from the Robuter after each trial. A walkman playing white noise (controlled by the subject on the experimenter's indications) masked the sound from the robot's servo motors and other possible sound cues, but could not prevent that almost all subjects could reliably distinguish trials "with robot" from trials "without robot". This did not have any fundamental consequences. The Robuter could move freely through the experimental room, constrained only by the cables driving the HMD and the response device (see below). These cables were attached to the Robuter's superstructure, and guided by the experimenter when necessary, so that the subjects could not infer directional information from them. Periodically, the Robuter had to be repositioned in the experimental room; this was always announced to the subject.

\section{Figure 2 ABOUT HERE.}

No direct form of synchronisation was possible between the physical and visual stimuli. Therefore, a trigger apparatus was developed that, using an infrared photodiode, detected the onset of the robot's displacement with a precision of not more than a millimetre. This information was conveyed via a serial link to the visual stimulus generator that had been busy-waiting for this signal. Upon arrival of the synchronisation signal, the movement of the optic flow generating dotscape (that was already visible) started no more than a single frame (1/60s) later. This system provided adequate synchronisation in almost all trials. Trials where the subject indicated that a noticeable delay had occurred were repeated at a later point during the session.

1 This deceleration is not sub-threshold, but was the lowest smooth deceleration we could attain, and much weaker than the preceding acceleration.

Visuo-vest. interaction in ego-motion reconstruction

René JV Bertin $5 / 24$ 


\section{Apparatus: visual stimulation}

The set-up of the visual stimulation and the response apparatus have been described elsewhere in detail (Bertin, Israël et al., 2000); we will repeat here only the essentials. Visual stimuli were generated on a Silicon Graphics O2 workstation, using the Performer 2.1 libraries. They consisted of "dotscapes" of white dots distributed on a horizontal ground plane in an otherwise dark (black) environment (a total of 3000 dots on a $25 \times 25 \mathrm{~m}$ plane). The position of the ground plane was calibrated to match the actual position of the ground as seen from the subject's position on the robot; approximately $1.25 \mathrm{~m}$ below eye-level on average. Optic flow corresponding to a given manoeuvre was generated by simulating movement of a virtual observer along that manoeuvre, using constant velocity (angular and tangential). As stated in the Introduction, we define a manoeuvre as a specific type of trajectory combined with a specific orientation (yaw) at any given point along that trajectory; yaw need not be yoked to the path. These displays were presented to the subject via an NVision Datavisor LCD HMD (FOV $48^{\circ}$ horizontal $\times 36^{\circ}$ vertical, 640x480 true VGA resolution at $60 \mathrm{~Hz}$ ). Stimuli were generated on the $\mathrm{O} 2$ in a $640 \times 480$ window that was captured by the O2's dedicated hardware, and sent as NTSC format S-Video via a Blackbox video-to-VGA converter to the HMD. (This extra step was necessary in order to preserve some screen "real estate" for the experimenter, and to create comparable conditions with earlier experiments.) Figure 3 shows time exposure snapshots of the optic flow generated by the different manoeuvres, together with top-down views of these manoeuvres (see also below).

\section{Figure 3 About Here.}

\section{Apparatus: response}

Subjects were required to reproduce the manoeuvre they had reconstructed from the optic flow. For this, they could manipulate a vehicle-like input device. A graphics tablet measured position and orientation of this device, and sent this information to the $\mathrm{O} 2$ where a separate programme processed and recorded it. For feedback, a stylised representation of the vehicle's current position and orientation, and its travelled path were shown in the HMD. Subjects were instructed to guide their reproduction by this information and to ignore proprioceptive information (they could not see their hands nor the tablet). Straight lines in a cross and a circle were provided to serve as templates for linear and circular trajectories ${ }^{2}$. Buttons on the model vehicle allowed the subject to erase unsatisfactory or garbled reproductions, and to save those that optimally represented their percept.

\section{Stimuli.}

Stimuli were either visual, or visuo-vestibular. The visual stimuli simulated passively travelled manoeuvres using optic flow. Three different types of manoeuvre were presented this way, with different sizes. The vestibular stimuli consisted of physical displacements, either linear or pure rotations-in-place. Each simulated manoeuvre was presented 1x purely visually (visual stimulus), and $1 \mathrm{x}$ in combination with a vestibular stimulus (visuo-vestibular stimulus; see also figure 3). All stimuli (conditions) were presented once only, aside from the exceptions mentioned below:

1. Tangential semicircles (the virtual observer always faced forward with respect to the simulated displacement: the conditions semicircle forward) were presented in 3 different radii: $R=1.5 \mathrm{~m}$, $R=3.25 \mathrm{~m}$ and $R=5 \mathrm{~m}$. (Because the angular velocity was fixed, tangential velocity increased proportionally with the radius.) The smallest radius conditions were presented twice ( $2 x$ visual, $2 x$

2 This information, and the vehicle's representation were of course shown only during the response phases!

Visuo-vest. interaction in ego-motion reconstruction

René JV Bertin $6 / 24$ 
visuo-vestibular; they were also included in a set of stimuli that we do not report on here). All rotations were clockwise: the pilot experiment (see below) had not revealed any leftward/rightward differences. In the visuo-vestibular trials, the subject was subjected to a forward linear physical motion as described above, because of the tendency to undershoot or not perceive the tangential component.

2. Outward semicircles (the virtual observer always faced $90^{\circ}$ outward with respect to the simulated displacement: the conditions semicircle outward) were presented with radius $R=1.5 \mathrm{~m}$ and $\mathrm{R}=5 \mathrm{~m}$. Clockwise and counter-clockwise rotations were presented. In the visuo-vestibular trials, subjects were subjected to a pure yaw physical motion in the appropriate direction, because of the undershoot of the angular velocity at especially the large radius.

3. Linear, forward movements with counter-clockwise $180^{\circ}$ yaw (the conditions linear half-turn) were presented with path lengths of $4.71 \mathrm{~m}$ and $7.85 \mathrm{~m}$. In the visuo-vestibular trials, subjects were subjected to a linear, forward physical motion, because the fact that the simulated displacement was along a straight path was not perceived.

All stimuli lasted for a total duration of $12 \mathrm{~s}$, with $2 \mathrm{~s}$ with stationary dotscape, then $8 \mathrm{~s}$ of simulated manoeuvre, and then again $2 \mathrm{~s}$ of stationarity.

\section{Experimental protocol.}

The experiment was performed in a darkened room, to prevent light from entering the HMD from behind. Subjects sat on the Robuter throughout the session, wearing the HMD. The conditions were presented in pseudo-randomised order. Before each visuo-vestibular trial, the robot's position was verified, the trigger device installed in an appropriate location, and the robot controller host was prepared. After each trial, the subjects shut off the walkman; the signal for this was the appearance of the "reproduction screen". They then reproduced the perceived manoeuvre as described above. In order to remove doubts about the intended response, we asked them to describe in a few words what they had just "drawn". When this description did not match the reproduction, they were asked to provide a better reproduction. Some additional questions were asked afterwards. When the reproduced manoeuvre represented a (more or less) circular movement, subjects were asked to qualitatively estimate the radius (small, medium, large, etc.), either by comparison with the previous stimulus, or directly. All subjects reliably detected the presence or absence of robot movement; nevertheless, we questioned systematically whether or not the robot had moved and, if so, how. In certain cases, more detailed questions were asked to relate specific particularities of the reproduction (e.g. a clear transition from linear to curvilinear movement) to the robot's movement. Finally, we asked whether or not a visuo-vestibular combination had been perceived as conflicting, or as "OK". Notes were taken, and the discussions were recorded for offline qualitative analyses and treatment of the reproductions.

Subjects were thoroughly instructed about this demanding protocol. It was stressed that they should principally concentrate on the visual stimulus, and that they should reproduce the manoeuvre that they had "perceived visually" (the radius estimation task was intended to help improve the focus on the visual stimulus). They were furthermore asked to indicate themselves when a noticeable delay had occurred between the onset of the vestibular and visual stimuli, and when they had sensed a strong conflict. Ample time was given to get comfortable with the model vehicle and reproduction task; during this training, comprehension of the possibly unyoked nature of path and orientation (yaw) was verified. Subjects were asked to draw their reproductions on as large a scale as possible. 
The experiment lasted between 1 and 2 hours, depending on the number of trials that had to be restarted.

\section{Data analysis.}

Artefacts in the reproductions were suppressed based on the verbal descriptions given, filtered with a lowpass filter and resampled to 20 points (19 segments of approximately equal length) as described in (Bertin, Israël et al., 2000). The stimulus top-down views in figure 3 (but not in figure 4) have been obtained with this same protocol.

\section{Figure 4 about Here.}

Responses were analysed using two different measures. The first measure describes each response in terms of three angles: the reproduced yaw $\left(\Psi_{\mathrm{o}}\right)$, rotation of the path $(\Psi \mathrm{p})$ and the average orientation relative to the path $(<\Phi r>)^{3}$; figure 4 . This measure allows us to describe observer rotation (yaw) independently from the eventual curvedness of the trajectory over which this observer is displaced (rotation of the path). The average path-relative orientation links these two angles to obtain a generalised but quantitative measure of the reproduced manoeuvre. Thus, a semicircle forward manoeuvre is described as $\left\{\Psi \mathrm{p} ; \Psi_{\mathrm{O}} ;\langle\Phi \mathrm{rr}\rangle\right\}=\left\{-180^{\circ} ;-180^{\circ} ; 0^{\circ}\right\}$. This measure is only fully defined for responses that are not rotations in place (RIP; for RIPs, only yaw is defined).

The second measure is a figural distance measure, adapted from (Conditt, Gandolfo et al., 1997). This measure quantifies the overlap (or rather: the error in the overlap) between a reproduction and the corresponding stimulus manoeuvre. It is a function of the spatial figural distance (Dfs: the average distance between the individual corresponding points on the trajectories) and the angular figural distance (Dfa: the average difference in orientation at these points, normalised with respect to Dfs). Here, we use $\mathrm{Df}=\sqrt{\mathrm{Dfs}^{2}+\mathrm{Dfa}^{2}}$. Thus, a perfect response would give a value of 0 (perfect overlap) according to this measure; any deviation will result in (increasingly) positive values. To obtain sensible comparisons among the stimulus/response differences, all responses were first shifted, rotated and/or scaled so as to obtain a maximum overlap with the corresponding stimulus (this was done by minimising the figural distance using a Simplex downhill method). This operation was possible because position, scale and orientation of the reproductions are not informative in our protocol: initial position and orientation were not defined, and absolute distance (scale) is not conveyed by the optic flow. The advantage of this figural distance measure over the other measures is that it can be applied to all responses (RIP or not), and that it captures other aspects as well: e.g. three orthogonal linear segments can correspond to a $180^{\circ}$ path rotation, while never being equal to a semicircle.

Statistical analyses were performed using the R data analysis language (http://www.r-project.org). The Df figural distance measure was log-transformed for analysis in order to improve normality.

Before starting the experiment just described, we conducted a pilot study, in which 9 of the current subjects (out of 17 total) took part, several weeks to several months before participating in the main experiment. In the pilot experiment, only visual stimuli were presented: the semicircle forward conditions described above, and rotations in place (both clockwise and counterclockwise). The experimental protocol was rigourously identical, except that subjects were seated on a regular chair instead of on the Robuter (and obviously no enquiries were made regarding the perception of vestibular stimuli).

\footnotetext{
3 NB: we use $\Psi$ to refer to rotation and $\Phi$ to refer to orientation. Thus, $\Phi$ o represents the observer's orientation at a given point, and $\Psi_{0}$ the amount of change in this orientation ("yaw") relative to the starting point.

Visuo-vest. interaction in ego-motion reconstruction

René JV Bertin 


\section{Results.}

Compared to our earlier visual experiments (Bertin, Israël, and Lappe, 2000), subjects performed in a highly similar fashion in the conditions in which only visual information was presented. We again observed many RIP responses in the smallest radius conditions. The perceived rotation of the subject (yaw) and of the path undershot the presented angles when the stimulus simulated a movement along a semicircle with tangential orientation (the semicircle forward conditions; see below). The linear half-turn conditions were in general perceived as tangential, curvilinear manoeuvres.

In order to test reproducibility (average and intra-subject) of the reconstructed manoeuvres, we had conducted the pilot experiment. Comparing the data from the pilot and from the main experiment, no significant differences were found in the various measures, neither for the subjects that participated in both experiments, nor between the subject groups as a whole. Furthermore, the results for the visual stimuli (from the pilot and the main experiment) were in good agreement with the results obtained in our earlier studies (with a highly similar protocol). This indicates that good reproducibility can be attained with our experimental protocol.

It is thus possible to use the obtained results to test our hypothesis that the final percept (the reconstructed manoeuvre) of our visual stimuli is largely determined by the initial percept. If this hypothesis is correct, then an initial vestibular stimulation that modifies the initial percept should modify the reconstructed manoeuvre. Improvement of the veracity of the initial percept, should lead to an improved veracity of the final reconstruction. We indeed observed such effects. Figure 5 shows all subjects' responses to one particular stimulus (semicircle forward, $\mathrm{R}=1.5 \mathrm{~m}$, purely visual (5a) and visuo-vestibular (5b) presentation).

\section{FiguRES 5 \& 6 ABOUT HERE}

\section{Improvement of visual perception by a vestibular stimulus (conditions semicircle forward)}

We imposed a linear physical displacement at the onset of visual stimuli simulating a displacement along a semicircular tangential path. We observed several positive effects of the vestibular stimulus on the perception of the visual stimulus. A striking effect was reduction (around 50\%) in the number of erroneous rotation in place perceptions in response to the smallest radius condition ( $\mathrm{p} \approx 0.013$, Kruskal-Wallis rank sum test over all radii). This is quantified in figure 6 (by the oblique lines; cf. figure 5). This was the result that we had expected.

The initial vestibular stimulus thus improved the perception of the translation simulated in the visual stimulus. The perceived manoeuvres were also significantly more veridical from a quantitative point of view when the vestibular stimulus was presented. We observed the following effects. Without the vestibular stimulus, the reproduced yaw tended to be around $18 \%$ smaller than the presented yaw (undershoot, showing as a positive error in figure 7a). The vestibular stimulus (a linear displacement!) reduced these errors to around $7 \%$ overshoot (ANOVA, $F(1,13)=10.12$; $\mathrm{p} \approx 0.0072$; figure $7 \mathrm{a}$ ). The effect was strongest and significant only in the small radius condition $(F(1,13)=10.35 ; \mathrm{p} \approx 0.0067)$, but there was no significant size/modality interaction.

Without a vestibular stimulus, the perceived rotation of the path also undershot the presented values: around $22 \%$ on average. With the vestibular stimulus the error was reduced to $6 \%$ overshoot (ANOVA, $\mathrm{F}(1,11)=8.912 ; \mathrm{p} \approx 0.012$; figure $7 \mathrm{~b}$ ). 
The perceived orientation relative to the path was also improved by the vestibular stimulus, that is, it was more tangential (figure 7c). Without a vestibular stimulus, there was around $47^{\circ}$ overshoot on average; subjects tended to reproduce manoeuvres with a progressively more outward orientation. With a vestibular stimulus, this error reduced to approximately $25^{\circ}$ overshoot (ANOVA, $\mathrm{F}(1,11)=3.73 ; \mathrm{p} \approx 0.08$, marginally significant).

The figural distance measure confirmed these positive effects: there was less difference between stimulus and reproduction when the vestibular stimulus was present (ANOVA, $F(1,13)=10.80$; $\mathrm{p} \approx 0.0059$ for $\log (\mathrm{FD})$; figure $7 \mathrm{~d}$ ).

\section{Figure 7 ABOUt Here}

\section{Adverse effect of a vestibular stimulus on visual perception (conditions semicircle outward)}

We also imposed a pure physical rotation in place at the onset of visual stimuli that simulated a displacement along a semicircular path with outward orientation. Without the vestibular stimulus, these visual stimuli were often perceived as lateral linear translations when the large radius version was presented (resulting in a significant $50 \%$ undershoot of the path-rotation; $t$-test, $\mathrm{p}<0.0012$ ). The small radius version was again often perceived as a rotation in place. We had expected to observe more curvilinear reproductions for the large radius with the vestibular stimulus than without. However, we did not find a significant improvement (the undershoot reduced to $33 \%$, still significant; $\mathrm{t}$-test, $\mathrm{p}<0.03$ ). Adding the vestibular stimulus actually increased the number of rotation in place responses from 39\% to 59\% (all radii; $\mathrm{p} \approx 0.038$, Kruskal-Wallis rank sum test), thus in fact deteriorating perception instead of making it more veridical.

\section{Absence of effect of the vestibular stimulus (conditions linear half-turn)}

Finally, we presented a linear vestibular stimulus with visual stimuli simulating a linear displacement with $180^{\circ}$ degree of yaw (full-body rotation). We had hoped to observe the perceived pathrotation to be closer to $0^{\circ}$ when an initial vestibular stimulus had been presented. Unfortunately, the perception of the visual stimulus was not more veridical with the vestibular stimulus than without. Without vestibular stimulus, the average perceived path-rotation was $118^{\circ}$ for the short and $221^{\circ}$ for the long version: the subjects perceived curvilinear paths as expected. With the vestibular stimulus, these figures are $155^{\circ}$ for the short and $296^{\circ}$ for the long trajectories (all highly significantly different from $0^{\circ}$ ). Thus, the reproduced manoeuvres remained predominantly curvilinear instead of linear, despite the vestibular stimulus.

\section{Discussion}

Optic flow is one of the important visual sources of information concerning ego-motion in space. It is however prone to misinterpretation when information is missing, under-represented or ambiguous: this can induce illusions. It has been shown that these illusions of the perceived heading direction can be reduced by adding additional visual but also non-visual information: a structured environment (Li and Warren, Jr., 2000), eye- or head movements (Crowell, Banks, Shenoy, and Andersen, 1998) and even whole-body tilt (Sibigtroth and Banks, 2001). In the present paper, we tested the hypothesis that the final reconstruction of passively travelled manoeuvres simulated with optic flow is governed by the initial percept, and can thus be altered by providing vestibular information at the visual stimulus' onset. We show that this hypothesis is confirmed. We also show that such an initial vestibular stimulus can improve the perception of the visual stimulus in a limited number of conditions. This is (to our knowledge) the first time that such effects of a vestibular stimulus on the perception of a visual stimulus have been reported. 
When we presented a linear vestibular stimulus with a visual stimulus that simulated movement along a circular arc with tangential orientation (semicircle forward), perception of the visual stimulus improved. We predicted that the vestibular stimulus would decrease the number of erroneous rotation in place (RIP) responses by increasing the saliency of the translation component of the simulated manoeuvre. This is indeed what we found: there were $50 \%$ less RIP responses in the small radius condition with vestibular stimulus than without vestibular stimulus. But, surprisingly, the simulated manoeuvres were also better perceived according to quantitative measures: the error in the perception of yaw decreased by almost $50 \%$, the error in the perception of the pathrotation somewhat less.

There are several possible explanations for this improvement. First of all, almost self-evident: perceiving the visual stimulus as a RIP and the vestibular stimulus as linear or curvilinear displacement would probably have induced a strong sense of conflict (that could be avoided by not perceiving RIPs). A second, more appealing explanation lies in the field of view. The high number of RIP responses in the vision-only conditions can most likely be explained by the limited field of view (FOV) of our HMD; it was approximately $40^{\circ}$ horizontally (fixed projection screens can attain $90^{\circ}$ or more, but could not be used for obvious reasons). When the FOV is decreased, radial components in the optic flow (corresponding to forward ego-translation) become less salient. Thus, for a small radius curvilinear tangential manoeuvre, the translation component of the simulated egomotion may become undetectable against the rotation component: a RIP response will be the result. The explanation for the observed improvement would then be that an initial physical, forward translation increases the saliency of the under-represented components: "visual translation" supported by "vestibular translation". A third explanation might lie in the side-effects provoked by the vestibular stimulus. A passive linear physical displacement is likely to cause some initial confusion (or surprise), but it lacks the lasting disorienting effect of a passive rotation. A limited amount of initial surprise or confusion could help (or oblige) the subject to concentrate on the interpretation of the visual stimulus (this would also explain the observed quantitative improvement), whereas a lasting disorientation will evidently have adverse effects.

Additional support for our hypothesis, and for the second explication above, comes from a stimulus combination in which the visual stimulus simulated a semicircular movement with outward orientation and the vestibular stimulus was a RIP. We predicted that this would increase the perception of the path's rotation in the large radius conditions. Instead, we found that the number of RIP responses increased, even for the large radius stimuli. Thus, a rotational vestibular stimulus increases the saliency of the rotational component in the visually simulated manoeuvre. This result supports the effect discussed above. However, in this case the perception of the visual stimulus is worse with than without vestibular stimulus: the translational component is often not (well) perceived. It is known that a strong vestibular stimulus can cause the temporary perceptual freezing of a moving visual pattern (Pavard and Berthoz, 1977; Buizza, Leger, Droulez, Berthoz, and Schmid, 1980), and recent studies found physiological evidence for reciprocal cross-modal inhibition (Wenzel, Bartenstein et al., 1996; Brandt, Bartenstein et al., 1998). Also, the vestibular stimulus we imposed can temporarily decrease the retinal flow by inducing a VOR eye movement in the same direction as the optic flow in the visual stimulus: this will strongly modify the retinal flow. The suppression of the translation component may be explained by a combination of central suppression and peripheral modification of the visual input.

Finally, we found that a vestibular stimulus that does not provide additional, helpful information has little or no effect. We imposed a linear vestibular stimulus at the onset of visual stimuli simulating a linear translation combined with a large $\left(180^{\circ}\right)$ yaw rotation, predicting that this would increase the linearity of the reproductions (alone, this visual stimulus usually results in reproduc- 
tions of curvilinear, tangential manoeuvres). This effect did not occur. We can understand this given the results from the semicircle forward conditions. When a semicircle forward visual stimulus is perceived as a tangential curvilinear manoeuvre, adding a linear physical forward displacement does not qualitatively alter that perception. The physical displacement may itself be perceived as slightly curvilinear, or it may slightly reduce the curvedness of the visually perceived movement, but it is not incompatible with that visual percept. Thus, when optic flow can be perceived as either a tangential curvilinear manoeuvre (illusion) or as a linear translation with yaw (correct), a linear vestibular stimulus does not help in choosing between these two alternatives. It cannot disambiguate that particular optic flow. This finding is in agreement with the study by Crowell et al. (Crowell, Banks, Shenoy, and Andersen, 1998) who found that a physical, passive whole-body rotation cannot disambiguate a similar optic flow. The information that their vestibular stimulus gives ("the body turns") is also in agreement with both interpretations.

On the level of individual responses, different perceptions of the visual and vestibular stimuli could be observed. Surprisingly, many subjects had a clear percept of two separate manoeuvres the "visual movement" and the robot's movement (a dissociation that recalls the transparent visual perception of 2 different movements). It is clear from the above discussion that there was an influence of the vestibular stimulus on the perception of the visual stimulus. This was also visible at the level of individual responses. Often, for example, a semicircle forward visual stimulus combined with its corresponding vestibular stimulus (a linear displacement) was described as a manoeuvre "starting straight ahead, and then gradually deviating to the right". If the robot's movement was then described as being purely linear, the subject had had the aforementioned "transparent" perception. But the robot's movement was also often described as "the same as the visual manoeuvre", proof that influence of the visual stimulus on the perception of the vestibular stimulus also occurred (in this example, it would have been a "mutual" interaction). Based on these observations, it is possible to predict the effect of vestibular stimuli that are not (perfectly) synchronised with the onset of the visual stimulus. Due to the time constants of the vestibular system, a vestibular stimulus that starts before the visual stimulus will be likely to exert an influence on the perception of the latter stimulus for a certain time. For example, we performed some trials in which the visual stimulus started when the physical displacement attained its highest speed. This gave results identical to those described in this paper. Administering the vestibular stimulus at a time $\mathrm{T}$ during the visual stimulus will of course very likely give different results. There will probably be an effect on the perception of the visual stimulus, if not only because the onset of physical motion would be the qualitative event that we postulate many subjects "wait" for in order to update their percept, "look with renewed interest" at what is happening in the visual display. Because of this, the effect of the visual stimulus (the perceived ego-motion at $\mathrm{T}$ ) on the perception of the vestibular stimulus will likely be small. The final percept of the visual stimulus should be quite different, showing a clear change around time $T$, if the vestibular stimulus caused a significant qualitative update of the percept. But nothing in our current data would lead us to expect that an ethically acceptable vestibular stimulus might retroactively improve the veracity of the already perceived part of the visual stimulus.

A word of caution is at place here about vestibular "impulses" and the time constants of the vestibular system. The vestibular system detects acceleration, but (roughly speaking) signals velocity: a form of integration, lowpass filtering, takes place already in the sensory organs itself. Thus, considerable time constants can be expected - and indeed they are. The result of this is that an "impulse" as we applied induces vestibular movement perception that persists until well after the peak in the acceleration and even velocity profile. In our experiment, this was not an insurmountable problem. The visuo-vestibular combinations were chosen to limit conflict. The rotatory vestibular stimuli are most likely to give rise to longer lasting perception of rotation, but were combined with one visual 
stimulus type only where the potential effect would not qualitatively change because of this. Also, in most cases cross-modal interaction reduced potential conflict arising during the stimulus, as described above. However, in applications different from ours vestibular time constants are of course an issue that should not be too lightly dealt with. The rather sharp deceleration in the linear stimuli just after the initial acceleration suggests that subjects may have perceived a change in direction. We tested this in a purely vestibular control experiment. Out of 6 subjects, only 1 perceived a reversion of direction towards the end of the stimulus:, a total of 3 out of 36 responses. In the visuo-vestibular experiment, this is unlikely to have occurred because of the cross-modal interactions mentioned earlier. And none of the subjects reported a reversion when asked how the robot had moved.

It has been common knowledge (used to good effect in the entertainment industry) that short vestibular stimuli can enhance sensations primarily produced by visual stimulation. Here we show for the first time that a short-lasting vestibular stimulus given at the onset of a longer-lasting visual stimulus can influence the perception of the latter in a navigation/orientation task more complex than instantaneously indicating the direction of heading. Our results suggest that cross-modal congruence is essential: a linear vestibular stimulus can improve the perception of translation in the visual stimulus, and a rotational vestibular stimulus can improve the perception of the rotational component in the visual stimulus. The resulting effect on visual perception can be positive (perception of a displacement together with rotation, instead of only rotation) but also negative (perception of only rotation, instead of a displacement together with rotation...). These findings can be of relevance to the development of low-cost driving simulators, especially those making use of HMDs for visual display. Our results suggest that physical rotation for augmenting realism may well have to be used with caution. Furthermore, the fact that dissociation occurs relatively often suggests that situations of (mild) visuo-vestibular conflict do not necessarily lead to immediate motion sickness. Dissociation, the concurrent existence of separate visual and vestibular percepts of ego-motion does of course not help a simulator's goal of improving salience and veracity of egomotion perception, but it may be interesting to study it as it may shed more light on the build-up of simulator sickness.

\section{Acknowledgements}

The authors wish to thank I. Israël, J. McIntyre and an anonymous reviewer for critically reading a previous version of this paper, and the many helpful, constructive comments that ensued. Michel Ehrette created the "vehicle"; France Maloumian most of figure 1. 


\section{References}

Bakker NH, Werkhoven PJ, Passenier PO (1999) The effects of proprioceptive and visual feedback on geographical orientation in virtual environments. Presence 8: 36-53

Berthoz A, Israël I, Georges-François P, Grasso R, Tsuzuku T. (1995) Spatial memory of body linear displacement: What is being stored? Science 269: 95-98

Bertin RJV, Israël I, Lappe M (2000) Perception of two-dimensional, simulated ego-motion trajectories from optic flow. Vision Res. 40: 2951-2971

Brandt T, Bartenstein P, Janek A, Dieterich M (1998) Reciprocal inhibitory visual-vestibular interaction. Visual motion stimulation deactivates the parieto-insular vestibular cortex. Brain 121 ( Pt 9): 1749-1758

Buizza A, Leger A, Droulez J, Berthoz A, Schmid R (1980) Influence of otolithic stimulation by horizontal linear acceleration on optokinetic nystagmus and visual motion perception. Exp.Brain Res. 39: 165-176

Conditt MA, Gandolfo F, Mussa-Ivaldi FA (1997) The Motor System Does Not Learn the Dynamics of the Arm by Rote Memorization of Past Experience. J.Neurophysiol. 78: 554-560

Crowell JA, Banks MS, Shenoy KV, Andersen RA (1998) Visual self-motion perception during head turns. Nature neuroscience 1: 732-737

Ehrlich SM, Beck DM, Crowell JA, Freeman TC, Banks MS (1998) Depth information and perceived self-motion during simulated gaze rotations. Vision Res 38: 3129-3145

Gibson JJ (1950) The perception of the visual world. Houghton Mifflin, Boston

Groen EL, Valenti Clari MSV, Hosman RJAW (2000) Psychophysical thresholds associated with the simulation of linear acceleration. AIAA 4294-4302

Harris LR, Jenkin M, Zikovitz DC (2000) Visual and non-visual cues in the perception of linear self motion. Exp.Brain Res. 135: 12-21

Hlavacka F, Mergner T, Bolha B (1996) Human self-motion perception during translatory vestibular and proprioceptive stimulation. Neurosci Lett 210: 83-86

Ivanenko, Y.P., Grasso, R., Israël, I., \& Berthoz, A. (1997). The contribution of otoliths and semicircular canals to the perception of two-dimensional passive whole-body motion in humans. J Physiol (Lond) 502 ( Pt 1), 223-233.

Jürgens R, Boß T, Becker W (1999) Estimation of self-turning in the dark: comparison between active and passive rotation. Exp.Brain Res. 128: 491-504

Kim NG, Turvey MT (1998) Visually perceiving heading on circular and elliptical paths. J Exp Psychol Hum Percept Perform 24: 1690-1704

Koenderink JJ (1986) Optic Flow. Vision Res 26: 161-180

Lambrey, S., Viaud-Delmon, I., Berthoz, A. (2002) Influence of a sensorimotor conflict on the memorisation of a path travelled in virtual reality. Brain Res Cogn Brain Res 14 (1): 177-186

Lappe M, Bremmer F, van den Berg AV (1999) Perception of self-motion from visual flow [Review]. Trends in Gognitive Sciences 3: 329-336

Li L, Warren WH, Jr. (2000) Perception of heading during rotation: sufficiency of dense motion parallax and reference objects. Vision Res. 40: 3873-3894

Loose R, Ayan T, Probst T (1999) Visual motion direction evoked potentials are direction specifically influenced by concurrent vestibular stimulation. Clinical Neurophysiology 110: 192-199 
Mergner T, Becker W (1990) Perception of horizontal self-rotation : multisensory and cognitive aspects. In: Warren R, Wertheim AH (eds) Perception and control of self-motion. Lawrence Erlbaum Ass., Hillsdale, pp 219-263

Mergner T, Schweigart G, Müller M, Hlavacka F, Becker W (2000) Visual contributions to human self-motion perception during horizontal body rotation. Arch Ital Biol 138: 139-166

Mesland, B. S. About horizontal self-motion perception... 1998. PhD thesis, Utrecht University, the Netherlands.

Pavard B, Berthoz A (1977) Linear acceleration modifies the perceived velocity of a moving visual scene. Perception 6: 529-540

Probst T, Loose R, King SK, Stott JR, Wist ER, Wright R (1996) Perception of direction of visual motion. II. Influence of linear body acceleration. Behav Brain Res 81: 147-154

Probst T, Loose R, Niedeggen M, Wist ER (1995) Processing of visual motion direction in the fronto-parallel plane in the stationary or moving observer. Behav Brain Res 70: 133-144

Redlick FP, Jenkin M, Harris LR (2001) Humans can use optic flow to estimate distance of travel. Vision Res. 41: 213-219

Royden CS, Crowell JA, Banks MS (1994) Estimating heading during eye movements. Vision Res 34: 3197-3214

Rushton SK, Harris JM, Lloyd MR, Wann JP (1998) Guidance of locomotion on foot uses perceived target location rather than optic flow. Curr Biol 8: 1191-1194

Sibigtroth, M. P. and Banks, M. S. Vestibular Stimulation in Heading Estimation. Presentation at VSS, 2001.

von der Heyde, M., Riecke, B. E., Cunningham, D. W., and Bülthoff, H. Visual-vestibular sensor integration follows a Max-Rule: Results from psychophysical experiments in Virtual Reality. Poster at the Tübinger Wahrnehmungskonferenz (TWK) 2001

Wann, JP, Schwapp, DK (2000) Why you should look where you steer. Nature neuroscience 3: 647-648

Wann JP, Swapp D, Rushton SK (2000) Heading perception and the allocation of attention. Vision Res. 40: 2533-2543

Wenzel R, Bartenstein P, Dieterich M, Danek A, Weindl A, Minoshima S, Ziegler S, Schwaiger M, Brandt T (1996) Deactivation of human visual cortex during involuntary ocular oscillations A PET activation study. Brain 119: 101-110 


\section{Figures (to be rescaled as convenient)}

Figure 1: Experimental set-up. The Robuter ${ }^{\mathrm{TM}}$; its controller host (an i486 PC computer running customary software under DOS) and the visual stimulus generator (an SGI O2 workstation running customary Performer software under Irix 6.3) driving an NVision Datavisor LCD HMD. The Robuter is controlled via a radio link. Synchronisation between physical and visual stimulation is on the onset of the robot's movement, using a trigger apparatus containing an infrared photo diode, attached to one of the O2's serial ports. During the reproduction phases, the subject manipulated a vehicle-like input device for the reconstruction of the perceived manoeuvres. Position and orientation of this model vehicle were recorded on the O2; a stylised view of it was presented in the HMD for feedback. Cues arising from wire pull were avoided by attaching the cables to the Robuter behind the subject.

Figure 2: physical displacement velocity profiles (vestibular stimuli).

a) Velocity profile of the linear displacements. The dark, fat trace shows the average and standard deviation of the individual profiles that are shown in light grey. Speed was calculated from the robot's position signal.

b) Velocity profiles of the angular displacements. Display as described for figure 2a. Positive speeds (angles) indicate counter-clockwise rotations, negative speeds (angles) clockwise rotations.

Figure 3: Visual stimuli: top-down views of the trajectories and optic flow snapshots. The top-down views show the trajectories and the observer orientation (indicated by arrows) at an arbitrary number of equidistant positions (the open circles), as explained by the inset (I). Scale of the top-down views is given by bars in the figure's left margin. The optic flow snapshots are taken after approximately $4 \mathrm{~s}$ (half-way the stimulus duration), and during approximately $0.3 \mathrm{~s}$. (The subjects only saw the optic flow - moving dots - and not the other information shown in this figure). The flow generated by the 3 different types of manoeuvre is shown, with in the left column the small/short versions and in the right column the large/long versions. In addition, the figure's left margin gives an abstract representation of the physical displacement that was started at the onset of the corresponding visuo-vestibular trials.

Top row: Conditions semicircle forward: tangential, circular movements in clockwise direction. The medium sized radius is not shown. The vestibular stimulus was a linear translation of the Robuter on which the subject was seated.

Middle row: Conditions linear half-turn: straight translations with $180^{\circ}$ yaw rotation in counter clockwise direction. The vestibular stimulus was a linear translation.

Bottom row: Conditions semicircle outward: circular movements with $90^{\circ}$ outward orientation. The flow snapshots are taken from clockwise movements, but would be identical for counter clockwise movements. The vestibular stimulus was a rotation in place, congruent with the direction of rotation simulated in the visual stimulus.

Figure 4: Explication of the indices used in the quantitative analyses. See text (Methods) for details. In this example (clockwise semicircle with counterclockwise yaw; not used in the experiments), yaw $\Psi_{\mathrm{O}}=180^{\circ}$, path-rotation $\Psi \mathrm{p}=-180^{\circ}$ and path-relative orientation (gaze) $<\Phi r>=179.7^{\circ} \pm 109.8^{\circ}$; figural distance $\mathrm{Df}=4.857$ with spatial figural distance $\mathrm{Dfs}=4.689$ and angular figural distance $\mathrm{Dfa}=1.264$ (for a path length of $15.70 \mathrm{~m})$. NB: points are placed at arbitrary locations on the trajectories in this figure! 
Figure 5: all responses to the small conditions semicircle forward, without (A) and with (B) vestibular stimulation. The reproduced manoeuvres are displayed as in figure 3, except that for clarity, the orientation at each point is shown by thin streamlines defining the subject's behind (conform the literature on animal trajectories). The large dark grey dots are rotation in place (RIP) responses, for which orientation is not shown. All responses were normalised to the longest response (thus no scale is shown), shifted to start in the origin, and rotated such that the average direction of the first segment (the average departure direction) is at $90^{\circ}$ (along the positive $\mathrm{Y}$ axis). RIP responses were not altered, but displaced to the periphery. The two large, outlined arrows indicate the original average departure direction (the larger arrow) and the original average initial orientation (at the starting point; the smaller arrow); the angle between these arrows gives the average initial path-relative orientation ( $\Phi \mathrm{r})$. A clear effect of the vestibular stimulus on the number of RIP responses can be seen (cf. figure 6), as well as on the average initial path-relative orientation (the panels are on identical scale).

Figure 6: effect of vestibular stimulation (a linear displacement) on the number of erroneous RIP responses in the semicircle forward conditions; there is a significant reduction $(p \approx 0.013$; Kruskall-Wallis rank sum test) of the number of rotation-in-place responses. This is clearly due mainly to an improvement of the perception of the smallest radius. Symbols show individual observations (1: RIP response; 0: not a RIP; a small amount of jitter separates the observations). RIP response fractions (1: all RIPs; 0: no RIPs) are shown by the oblique lines. Vis: visual-only trials; VisVest: visuo-vestibular trials.

Figure 7: quantitative effects of vestibular stimulation on the perception of the semicircle forward stimuli. The effects on perceived yaw $\left(\Psi_{\mathrm{O}}\right)$ and path rotation $(\Psi \mathrm{p})$ are shown using relative errors (the error divided by the presented amount of rotation). The significance of the main effect is shown above each panel. Box-and-whisker plots are used: big dots indicate the median, thick lines connect the means, the boxes show the interquartile range, the whiskers 1.5 times the interquartile range and open dots indicate outliers.

a) effect on the perceived yaw. There is a significant reduction of the error to close to 0 over all conditions when a vestibular stimulus is presented.

b) effect on the perceived rotation of the path. The decrease in error with vestibular stimulation is highly significant.

c) effect on the perceived path-relative orientation. Presence of a vestibular stimulus (a linear displacement) causes the perceived path-relative orientation to be more tangential (=veridical) in all cases, but this effect is only marginally significant.

d) effect on the figural distance measure (the logarithm of the "distance" between stimulus and response). There is a highly significant increase in stimulus/response resemblance (log(FD) becomes more negative) for the visuo-vestibular conditions. 
Figure 1:

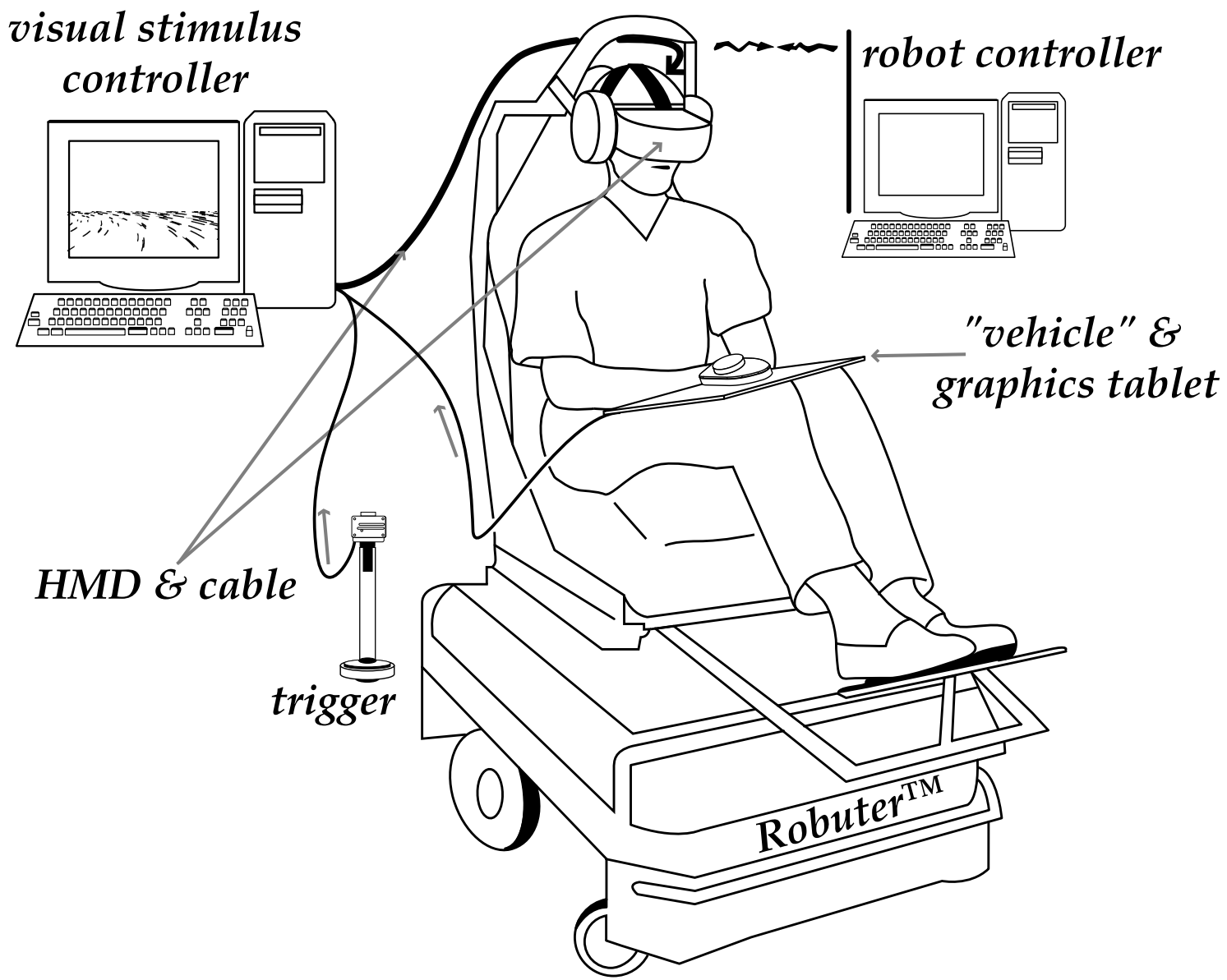


Figure 2a, b:

velocity $[\mathrm{m} / \mathrm{s}]$

Translation
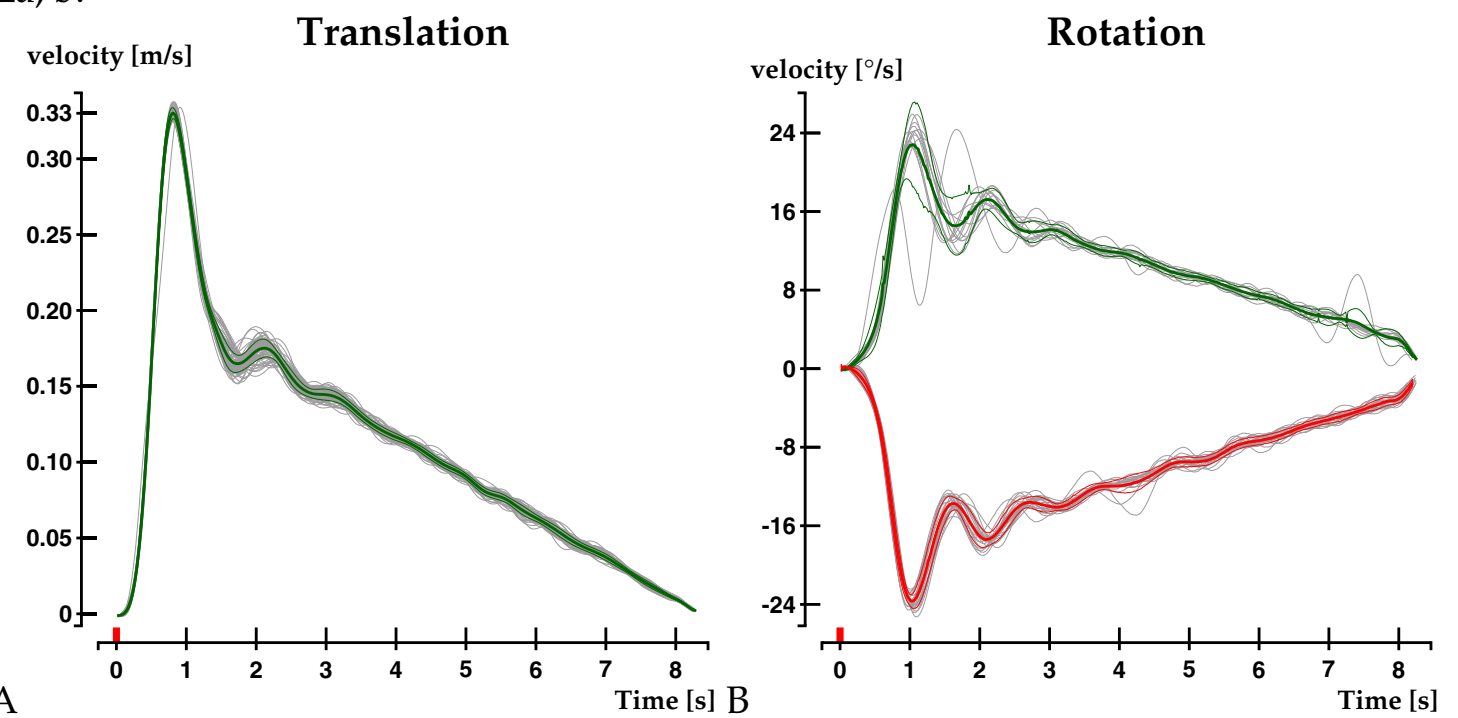
Figure 3:
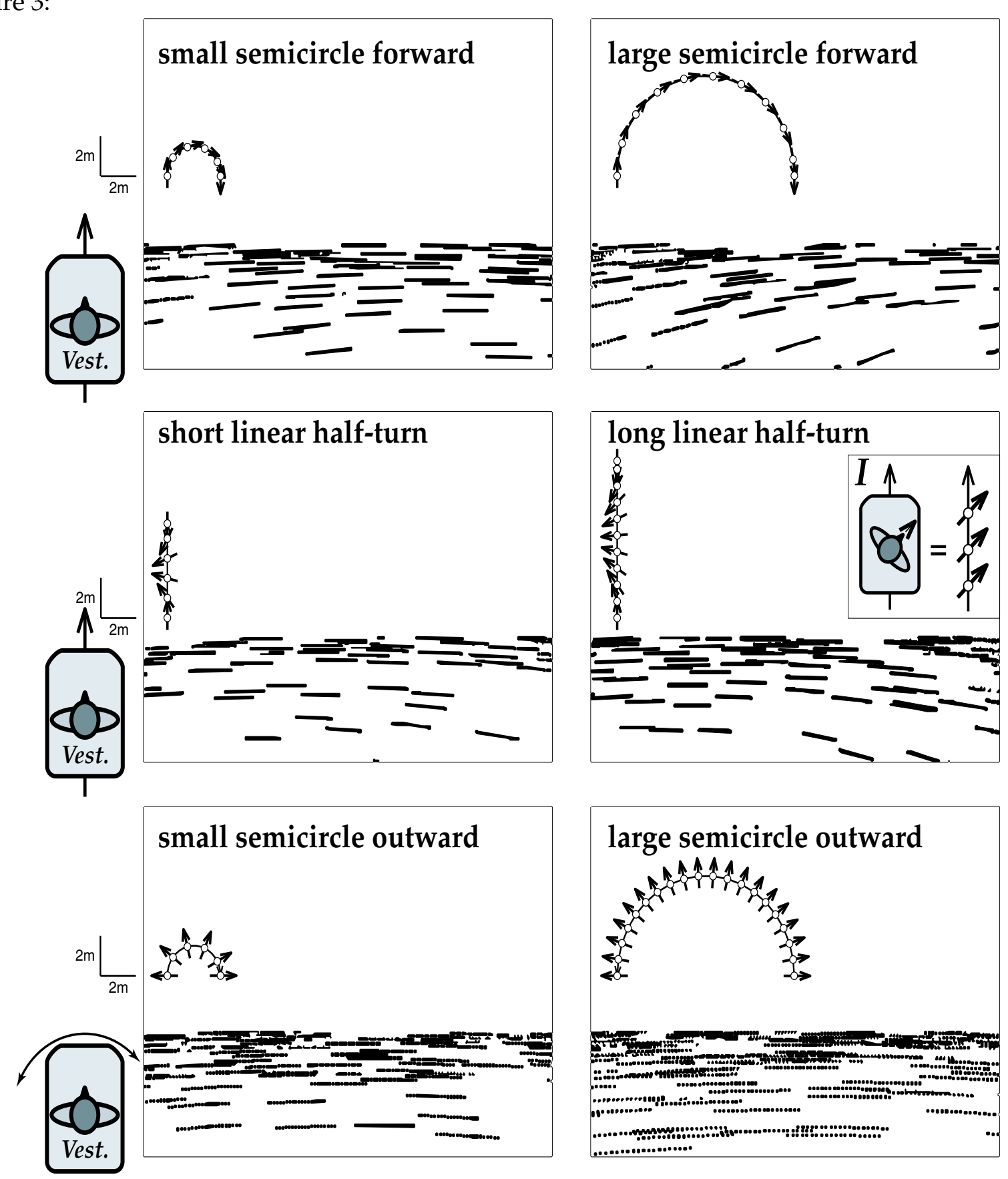
Figure 4:

Position: Y

Average orientation relative to path: $\langle\Phi \mathbf{r}\rangle$ $\varphi=\Phi p(\mathrm{i})-\Phi \mathrm{p}(\mathrm{i}-1)$
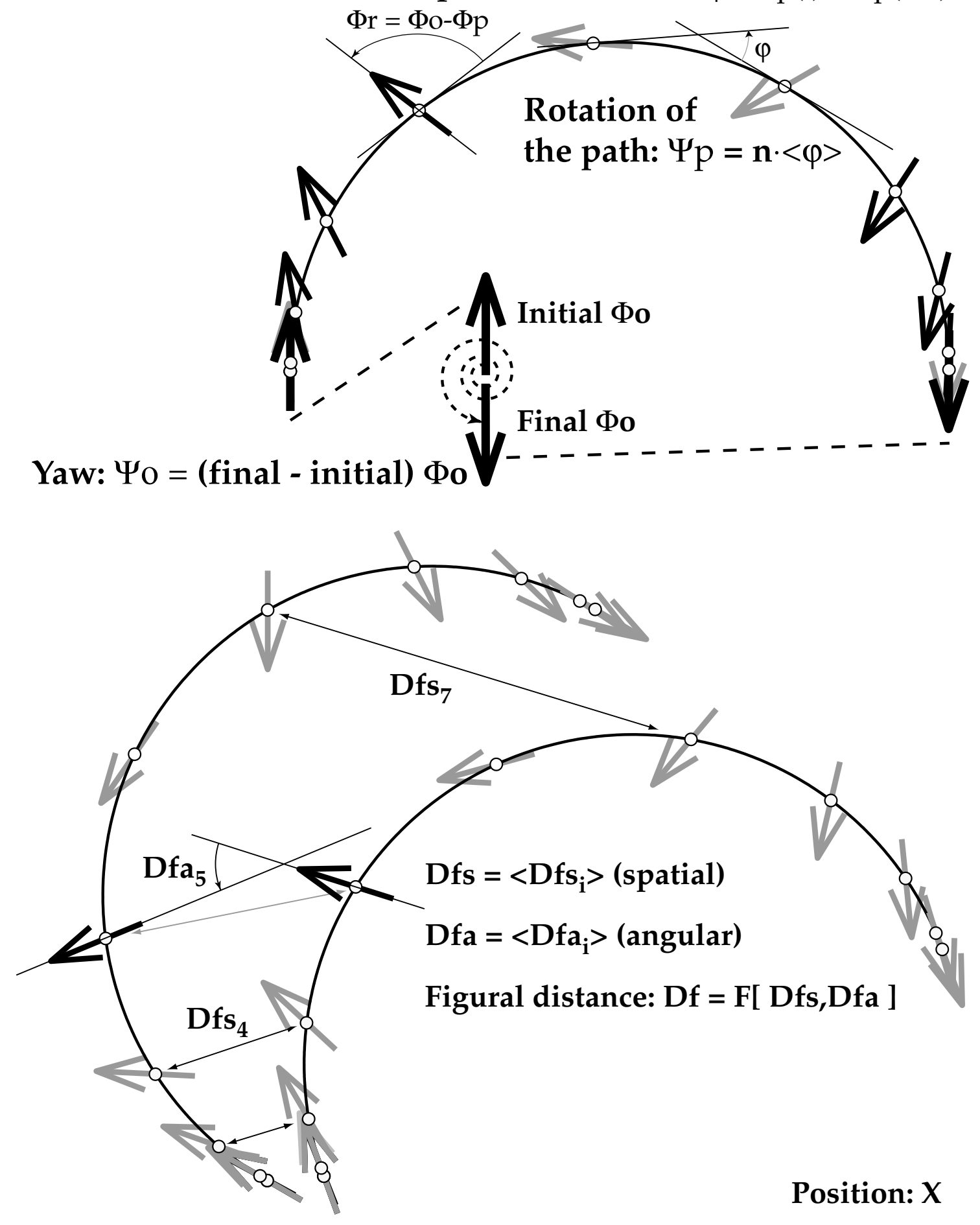
Figure 5:
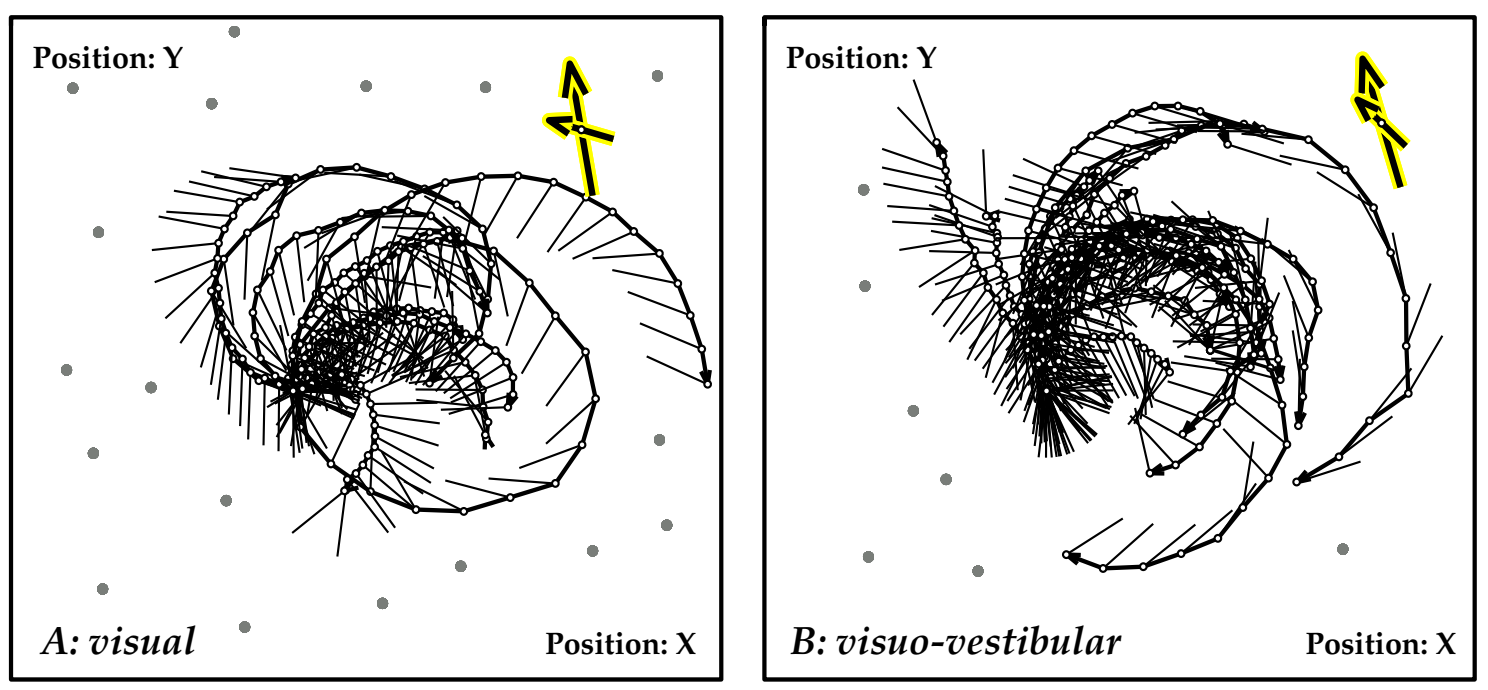
Figure 6:

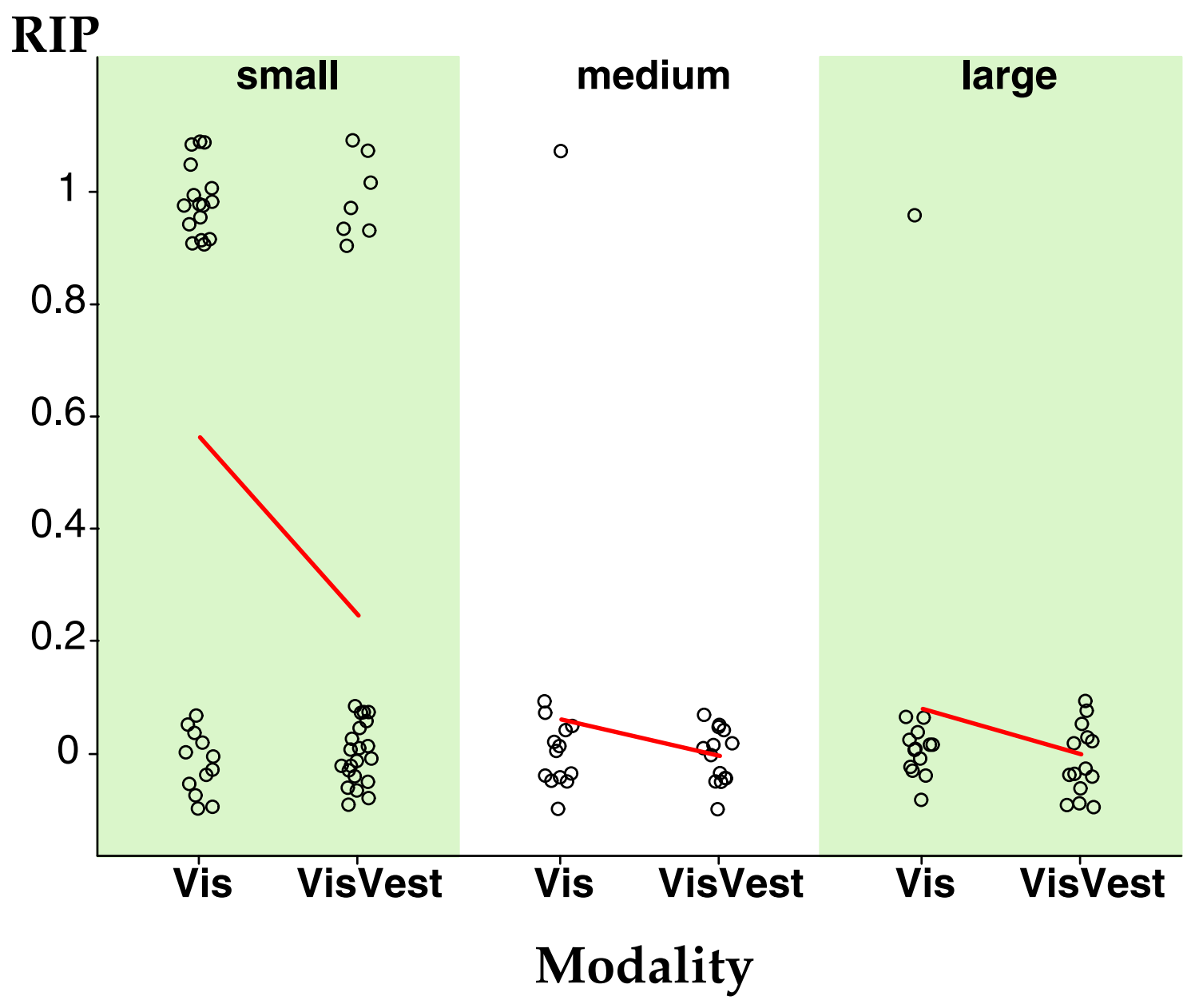


Figure 7:

Yo rel. error main effect: $F(1,13)=10.12 ; p=0.0072$
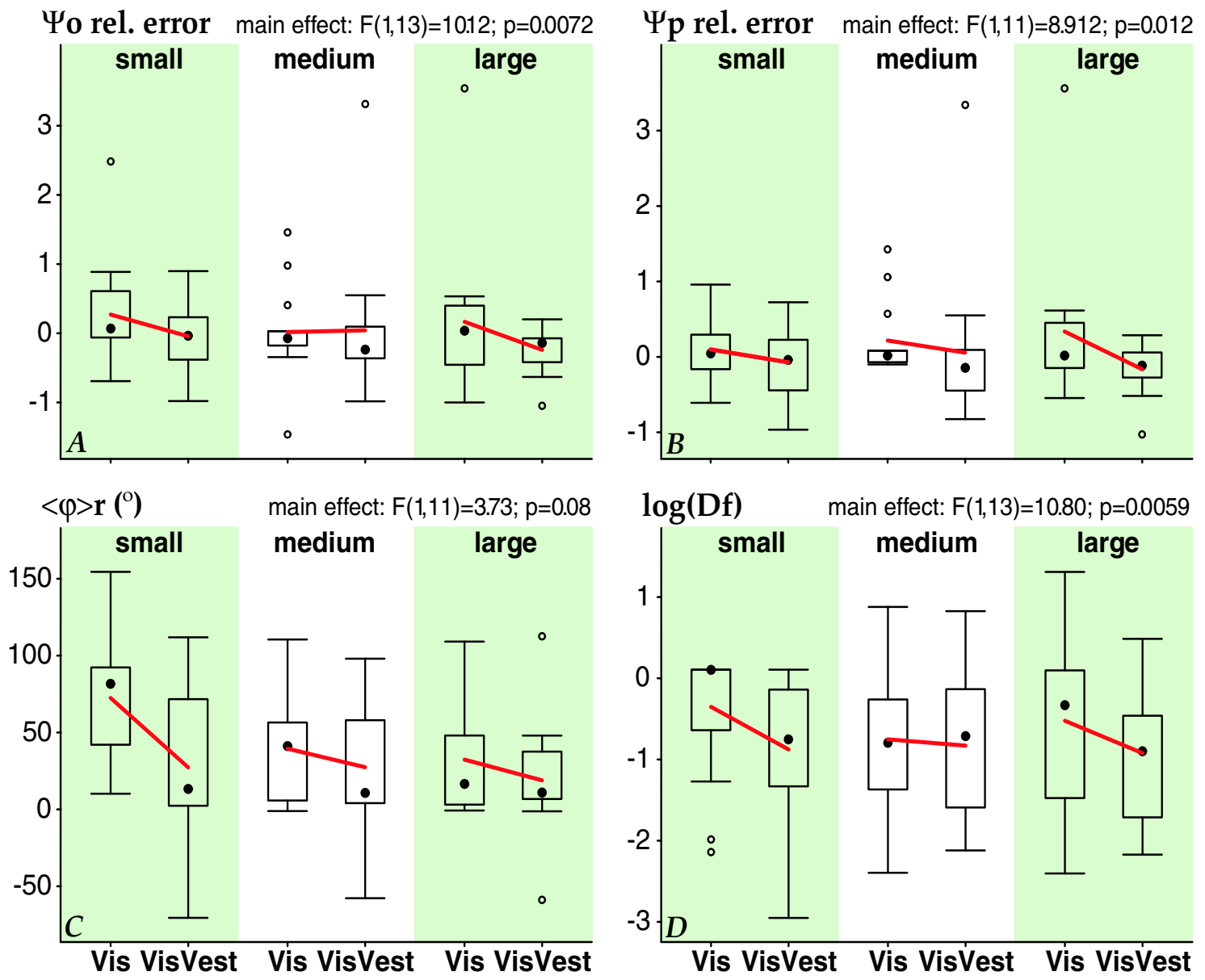

Modality 